\title{
Nyvad Criteria for Caries Lesion Activity and Severity Assessment: A Validated Approach for Clinical Management and Research
}

\author{
Bente Nyvad Vibeke Baelum \\ Department of Dentistry and Oral Health, Aarhus University, Aarhus, Denmark
}

\section{Keywords}

Caries management · Lesion activity · Lesion severity · Nyvad criteria. Prognosis

\begin{abstract}
The Nyvad classification is a visual-tactile caries classification system devised to enable the detection of the activity and severity of caries lesions with special focus on low-caries populations. The criteria behind the classification reflect the entire continuum of caries, ranging from clinically sound surfaces through noncavitated and microcavitated caries lesions in enamel, to frank cavitation into the dentin. Lesion activity at each severity stage is discriminated by differences in surface topography and lesion texture. The reliability of the Nyvad criteria is high to excellent when used by trained examiners in the primary and permanent dentitions. The Nyvad criteria have construct validity for lesion activity assessments because of their ability to reflect the well-known caries-controlling effect of fluoride. Predictive validity was demonstrated by showing that active noncavitated lesions are at higher risk of progressing to a cavity or filled state than do inactive noncavitated lesions. Lesion activity assessment performed successfully as a screening tool to identify individuals with a poor caries prognosis. Because of their predictive validity, the Nyvad criteria are superior to other current
\end{abstract}

caries lesion descriptors for the detection of changes in the lesion activity status over time. The Nyvad criteria fulfill all the formal requirements for a robust caries lesion classification and are recommended for evidence-based caries management in clinical practice and in research.

C 2018 S. Karger AG, Basel

Over the past decades, several sets of diagnostic criteria have been promoted for the classification of dental caries [this issue]. Different approaches may have made it difficult for dental practitioners to decide on a suitable caries classification to be used in dental practice. Such new classifications are often endorsed without being thoroughly evaluated for their advantages and disadvantages, and practitioners are not always trained in appraising the different systems. Which are the most important properties of a clinically relevant caries classification? Should the practitioner look for a classification that is very precise in estimating lesion depth, or should

This paper is based on a presentation given at the ORCA Saturday Afternoon Symposium "Critical Appraisal of Current Clinical Caries Diagnostic Systems" in Athens on July 6, 2016.

\section{KARGER}

(๑) 2018 S. Karger AG, Basel

E-Mail karger@karger.com

www.karger.com/cre
Prof. Bente Nyvad, $\mathrm{PhD}$, Dr Odont

Department of Dentistry and Oral Health, Aarhus University

Vennelyst Boulevard 9

DK-8000 Aarhus C (Denmark)

E-Mail nyvad@odont.au.dk 
Table 1. Description of the Nyvad criteria for caries lesion activity and severity assessment [Nyvad et al., 1999]

\begin{tabular}{|c|c|c|}
\hline Score & Category & Criteria \\
\hline 0 & Sound & Normal enamel translucency and texture (slight staining allowed in otherwise sound fissure) \\
\hline 1 & $\begin{array}{l}\text { Active caries } \\
\text { (intact surface) }\end{array}$ & $\begin{array}{l}\text { Surface of enamel is whitish/yellowish opaque with loss of luster; feels rough when the tip of the } \\
\text { probe is moved gently across the surface; generally covered with plaque } \\
\text { No clinically detectable loss of substance } \\
\text { Smooth surface: caries lesion typically located close to gingival margin } \\
\text { Fissure/pit: intact fissure morphology; lesion extending along the walls of the fissure }\end{array}$ \\
\hline 2 & $\begin{array}{l}\text { Active caries } \\
\text { (surface discontinuity) }\end{array}$ & $\begin{array}{l}\text { Same critera as score } 1 \\
\text { Localized surface defect (microcavity) in enamel only } \\
\text { No undermined enamel or softened floor detectable with the explorer }\end{array}$ \\
\hline 3 & $\begin{array}{l}\text { Active caries } \\
\text { (cavity) }\end{array}$ & $\begin{array}{l}\text { Enamel/dentin cavity easily visible with the naked eye; surface of cavity feels soft or leathery on } \\
\text { gentle probing } \\
\text { There may or may not be pulpal involvement }\end{array}$ \\
\hline 4 & $\begin{array}{l}\text { Inactive caries } \\
\text { (intact surface) }\end{array}$ & $\begin{array}{l}\text { Surface of enamel is whitish, brownish or black } \\
\text { Enamel may be shiny and feels hard and smooth when the tip of the probe is moved gently across } \\
\text { the surface } \\
\text { No clinically detectable loss of substance } \\
\text { Smooth surface: caries lesion typically located at some distance from gingival margin } \\
\text { Fissure/pit: intact fissure morphology; lesion extending along the walls of the fissure }\end{array}$ \\
\hline 5 & $\begin{array}{l}\text { Inactive caries } \\
\text { (surface discontinuity) }\end{array}$ & $\begin{array}{l}\text { Same criteria as score } 4 \\
\text { Localized surface defect (microcavity) in enamel only } \\
\text { No undermined enamel or softened floor detectable with the explorer }\end{array}$ \\
\hline 6 & $\begin{array}{l}\text { Inactive caries } \\
\text { (cavity) }\end{array}$ & $\begin{array}{l}\text { Enamel/dentin cavity easily visible with the naked eye; surface of cavity may be shiny and feels } \\
\text { hard on probing with gentle pressure } \\
\text { No pulpal involvement }\end{array}$ \\
\hline 7 & Filling (sound surface) & \\
\hline 8 & Filling + active caries & Caries lesion may be cavitated or noncavitated \\
\hline 9 & Filling + inactive caries & Caries lesion may be cavitated or noncavitated \\
\hline
\end{tabular}

the classification reflect the best treatment options available for different types of carious lesions?

The caries decline observed in many countries over the past 30 years poses further challenges in caries diagnosis. Because of the slower rate of progression of caries in many populations and a concomitant decrease in the number of cavities, it is no longer sufficient to score caries according to the WHO criteria [WHO, 1997]. Carious lesions can and should be identified and controlled at the noncavitated level, i.e., prior to the development of cavity formation [Nyvad and Fejerskov, 1997, 2015]. However, noncavitated lesions may differ widely by their risk of progression [Nyvad, 2004]. An up-to-date caries classification must, therefore, also provide information about the prognosis and current best management options for such noncavitated lesions.

In this review, we demonstrate that the Nyvad criteria for caries lesion activity and severity assessment [Nyvad et al., 1999] offer a validated approach for this purpose. We shall show that such criteria are not only useful for dental practitioners when performing caries control for their patients in daily practice, but also for researchers and dental public health care planners when estimating the effects of, or the need for, caries-controlling interventions.

\section{The Pathobiological Basis for the Nyvad Criteria}

The Nyvad classification is a visual-tactile caries classification [Nyvad et al., 1999]. The criteria were developed to reflect the entire continuum of caries lesion development, ranging from clinically sound surfaces, through noncavitated and microcavitated enamel caries lesions, to frank cavitation into the dentin. A unique feature of the classification is that in addition to assessing the 
severity of lesions (presence or absence of cavity formation), each severity score includes an assessment of lesion activity. The criteria are, therefore, designed to reproduce all the dynamic caries lesion transitions that may occur in a patient over time, either naturally or in response to caries control procedures.

Lesion activity is detected by assessment of the surface topography and texture of the lesions; an actively progressing lesion in enamel is matte and rough on gentle probing with the tip of a sharp explorer, while an inactive lesion appears shiny and smooth on gentle probing [Thylstrup et al., 1994]. These surface phenomena reflect the demineralization activity of the dental biofilm and may be observed visually. Rough surfaces of active noncavitated enamel lesions appear lusterless to the naked eye due to scattering of light while surfaces of inactive lesions appear shiny because of specular reflection [Nyvad et al., 2015]. Table 1 provides a detailed clinical description of the individual Nyvad scores [Nyvad et al., 1999].

Active dentin and root surface caries lesions are invaded by bacteria subsequent to demineralization [Nyvad and Fejerskov, 1990]. This explains why actively progressing dentin caries lesions are soft on gentle probing. However, prolonged tooth brushing with fluoride toothpaste may change the texture of a soft active dentin lesion into a leathery or hard inactive lesion [Nyvad and Fejerskov, 1986; Hansen and Nyvad, 2017]. This concurs with mineral uptake in the surface layer [Nyvad et al., 1997]. Some root/dentin lesions also take up stain, but lesion color does not help to discriminate lesion activity (Table 1).

Typical clinical examples of active and inactive caries lesions and their Nyvad scores are shown in Figure 1.

\section{Clinical Use of the Nyvad Criteria}

A proper diagnosis according to the Nyvad criteria requires clean and dry teeth. Otherwise, the surface topographical features of the enamel may be difficult to assess correctly. The presence of sticky biofilm adhering to a chalky/matte enamel lesion is strongly indicative of lesion

Fig. 1. Typical clinical manifestations of active and inactive caries lesions according to the Nyvad criteria [Nyvad et al., 1999]. Active noncavitated lesion on smooth surface (a) and occlusal surface (b). Inactive noncavitated lesion on smooth surface (c) and occlusal surface (d). Active (e) and inactive (f) lesion with microcavity on occlusal surface. Active (g) and inactive (h) cavitated lesion. a, b, d, e, f Reproduced with permission of S. Karger AG. c, g, h Reproduced with permission of Wiley Blackwell.

Nyvad Criteria for Caries Activity and Severity Assessment
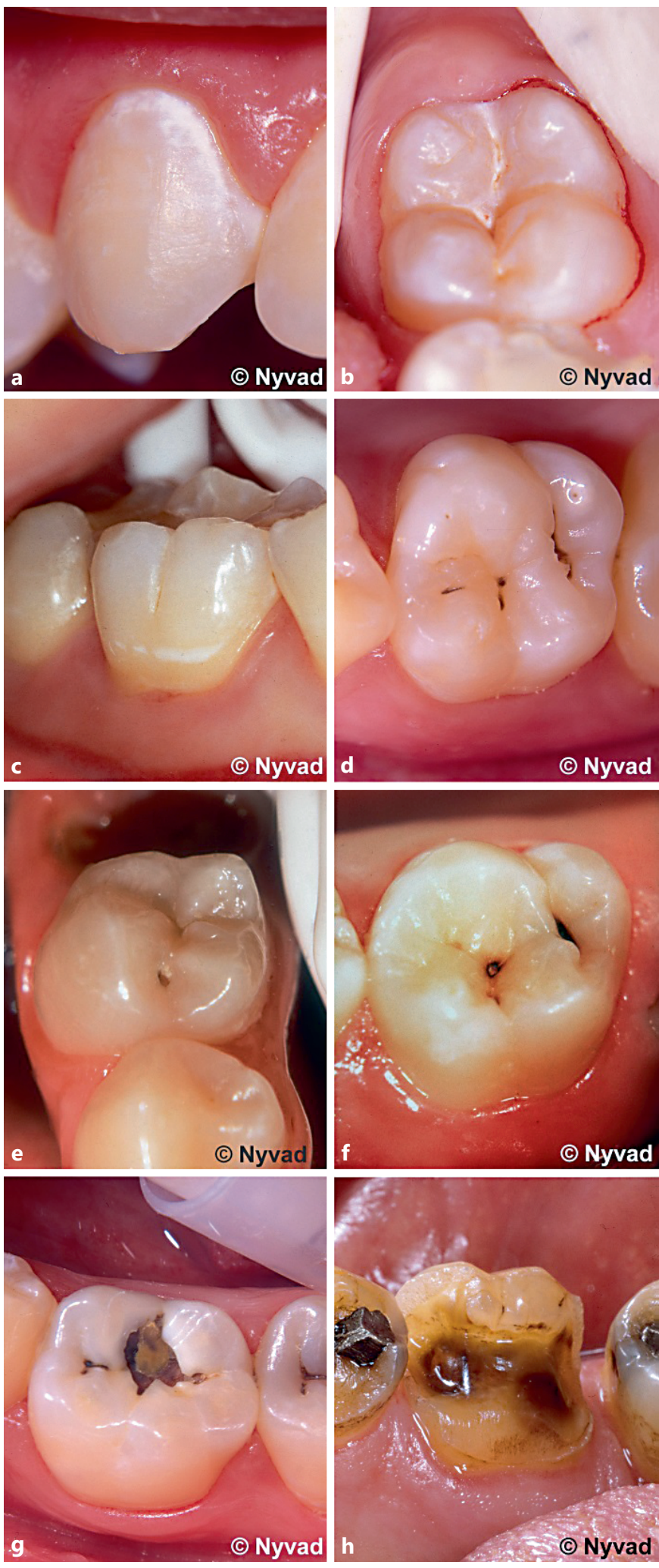

Caries Res 2018;52:397-405

DOI: $10.1159 / 000480522$ 
activity. Therefore, professional cleaning of the teeth is undesirable prior to a caries examination. During the examination, biofilm is removed from the tooth surfaces by the side of a sharp explorer while the tip of the explorer gently runs across the lesion to assess surface roughness. The explorer is never used to poke vigorously into the tissue to avoid damage to the surface layer of a lesion. Plastic and ball-ended explorers are not recommended as they may be incapable of conferring the necessary tactile "feel" of the surface features of the lesion. Indeed, probing is not necessary when the visual manifestation of activity is obvious.

Occasionally, it may be difficult to decide whether a lesion is active or inactive. The lesion may contain elements of both lesion activity categories, or the lesion may be at a transitional stage between active and inactive. From a treatment perspective, it is important not to overlook an active lesion. Therefore, a lesion should be recorded as active in all cases when any part of the lesion reveals the classical signs of activity. A detailed description of the examination procedures is given elsewhere [Nyvad et al., 2015], just as the differential diagnostic considerations are described by Nyvad et al. [2009] and Machiulskiene et al. [2009].

It should be appreciated that the Nyvad criteria cover the full clinical spectrum of caries lesion activity and severity in just 10 individual scores (scores from 0 to 9 ) (Table 1). These scores include separate codes for sound surfaces, fillings, and active and inactive secondary caries. A full-mouth examination typically takes no more than 5-10 min [Nyvad et al., 1999; Séllos and Soviero, 2011]. The criteria are user friendly and may easily be adapted to statistical analysis.

\section{Reliability of the Nyvad Criteria}

For any caries classification, it is important that the criteria are reproducible between and within examiners, since this is an indication of their ease of use. The reliability of the Nyvad criteria has been shown to be high to excellent when used by trained examiners under epidemiological conditions in both the primary and permanent dentitions [Machiulskiene et al., 1998; Nyvad et al., 1999, 2009; Braga et al., 2010; Séllos and Soviero, 2011; Tikhonova et al., 2014]. The reliability of the criteria is comparable to that of other common caries lesion descriptors not involving activity assessment [de Amorim et al., 2012; Fereira Zandoná et al., 2012]. This is remarkable because "subjective" lesion assessment is sometimes considered unreliable because activity cannot be quantified [Ando et al., 2017].

A closer analysis of the reliability data of Nyvad et al. [1999] revealed no indication of systematic intra- or interexaminer deviations. Most misclassifications (ca. 80\%) involved disagreement between the sound surface category and the noncavitated carious lesion (active or inactive) categories. These results corroborate earlier observations [Ismail et al., 1992; Séllos and Soviero, 2011] that diagnosis of noncavitated carious lesions is often mixed up with sound surfaces. Disagreements between the sound surface category and the noncavitated active or noncavitated inactive lesion categories occurred with about the same frequency (ca. 30\% of all misclassifications). Only some $10 \%$ of the misclassifications involved disagreement between noncavitated active and noncavitated inactive lesions. Adding lesion activity assessment does not reduce the reliability of caries diagnostic decisions including noncavitated diagnoses.

\section{Validity of the Nyvad Criteria}

A caries classification must be valid, i.e., it must measure what it purports to measure [Last, 2001]. The Nyvad classification includes aspects of both severity (progression stage) and activity of caries lesions, and assessment of the validity of the criteria must, therefore, include both aspects.

Braga et al. [2010] estimated the concurrent validity of the Nyvad criteria by comparing the clinical scores with the histological findings and found a strong correlation between clinical scores and histological lesion depth in occlusal surfaces of primary molars, similar to that found for ICDAS-II [Ismail et al., 2007] when combined with an adjunct system for lesion activity assessment (ICDASLAA). This is not surprising as both diagnostic systems are based on rather similar staging of severity. The authors further applied a $\mathrm{pH}$ indicator to histological sections of a subset of the extracted teeth to create a "gold standard" for lesion activity. Although a "substantial" correlation was observed between the clinical ICDASLAA scores and the "gold standard" activity scores, it was also clear that the Nyvad criteria had substantially larger specificity than the ICDAS-LAA for essentially similar sensitivity values. Nonetheless, a proper validation of caries lesion activity assessments requires observations from longitudinal studies or randomized clinical trials [Baelum et al., 2006]. 
We investigated the validity of the Nyvad criteria for lesion activity by 2 different methods based on data on caries lesion transitions observed in a clinical trial of supervised brushing with fluoride toothpaste [Machiulskiene et al., 2002]. One analysis showed that the Nyvad criteria had construct validity for lesion activity because the lesion transitions reflected the hypothetical cariescontrolling effect of fluoride [Fejerskov et al., 1981; ten Cate and Featherstone, 1996]. We could thus demonstrate the well-known inhibitory effect of fluoride on caries lesion progression (i.e., transitions from the inactive to the active state at all stages of lesion severity) and at the same time enhanced lesion regression (i.e., transitions from the active to the inactive state) [Nyvad et al., 2003].

In the other analysis, we tested the predictive validity of the criteria; i.e., whether different diagnostic categories were able to predict different outcomes of the caries process. The predictive validity of lesion activity assessment was confirmed by showing that active noncavitated lesions were at greater risk of progressing to a cavity or a filling than were inactive noncavitated lesions [Nyvad et al., 2003]. The effect was more marked in the control group than in the fluoride group, which had received supervised daily toothbrushing on school days for 3 years [Machiulskiene et al., 2002]. The predictive validity of the Nyvad criteria is of crucial interest from a caries management point of view. Overall, an active noncavitated lesion ran a $24 \%$ higher risk of progressing into a cavity/filling than an inactive noncavitated lesion over 3 years. Ignoring treatment of active noncavitated lesions by nonoperative interventions would, therefore, be inappropriate.

Interestingly, the Nyvad classification has also been validated for its ability to identify individuals with a poor caries prognosis. In a Finnish study, children were enrolled in a caries-preventive trial based on screening for the presence of at least 1 active noncavitated caries lesion using the Nyvad criteria [Hausen et al., 2007]. After an average of 3.4 years, the intervention group receiving intensified preventive interventions had developed $44 \%$ fewer cavities/fillings than the control group receiving standard treatment. This result was surprising since some years earlier the same group of authors had experienced poor effects of a high-risk strategy for the control of caries. However, in that study, participants were recruited based on caries risk factors, excluding factors such as the presence of active disease [Hausen et al., 2000]. Collectively, these observations emphasize the potential of the Nyvad criteria to target individuals that are at high need of caries-controlling interventions.

Nyvad Criteria for Caries Activity and Severity Assessment

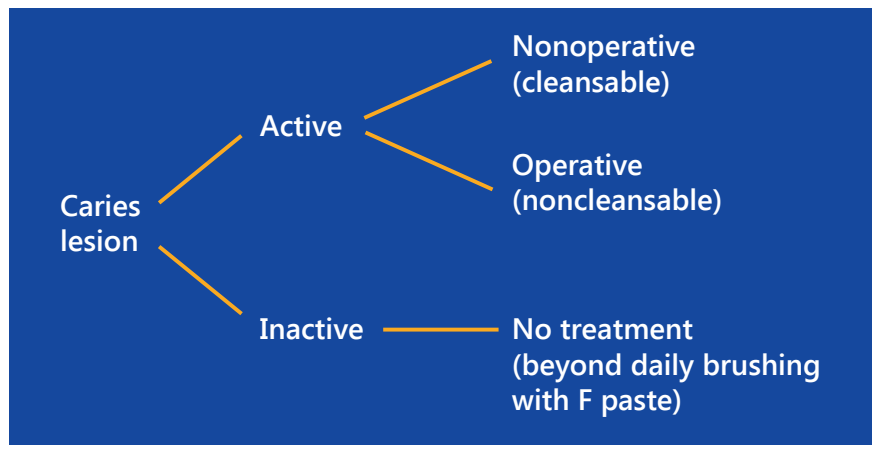

Fig. 2. Decision tree for treatment of caries based on lesion activity assessment.

\section{Usefulness of the Nyvad Criteria}

The scientific knowledge obtained by studying the properties of the Nyvad criteria suggests that such criteria can have a broad spectrum of applications in clinical caries management and research.

\section{Caries Control in Daily Clinical Practice}

Because of the predictive validity, the Nyvad criteria are a highly useful tool in the management of caries lesions, both at the level of the individual lesion and at the level of the individual patient. Such a refined tool is particularly beneficial in low-caries populations where most of the caries experience is represented by noncavitated stages of caries. By identifying active lesions during a clinical caries examination, the dentist will be able to direct professional management towards lesions that run the highest risk of progression, and the effect of interventions on such lesions may subsequently be monitored over time. A simple decision tree is helpful to illustrate this principle (Fig. 2). Active noncavitated lesions that can be cleaned by the patient should be treated by intensified nonoperative interventions according to individual needs (e.g., by oral hygiene instruction and additional use of topical fluorides), whereas inactive lesions need no additional intervention beyond basic prevention using daily brushing with fluoridated toothpaste. This decision rule may also apply to microcavitated active lesions that are easy to clean. Numerous clinical studies have demonstrated that such nonoperative strategies can be very efficient in caries control [for a review, see Nyvad and Fejerskov, 2015]. However, once an active cavitated lesion extends into dentin whereby cleaning has become difficult, operative treatment is needed. 


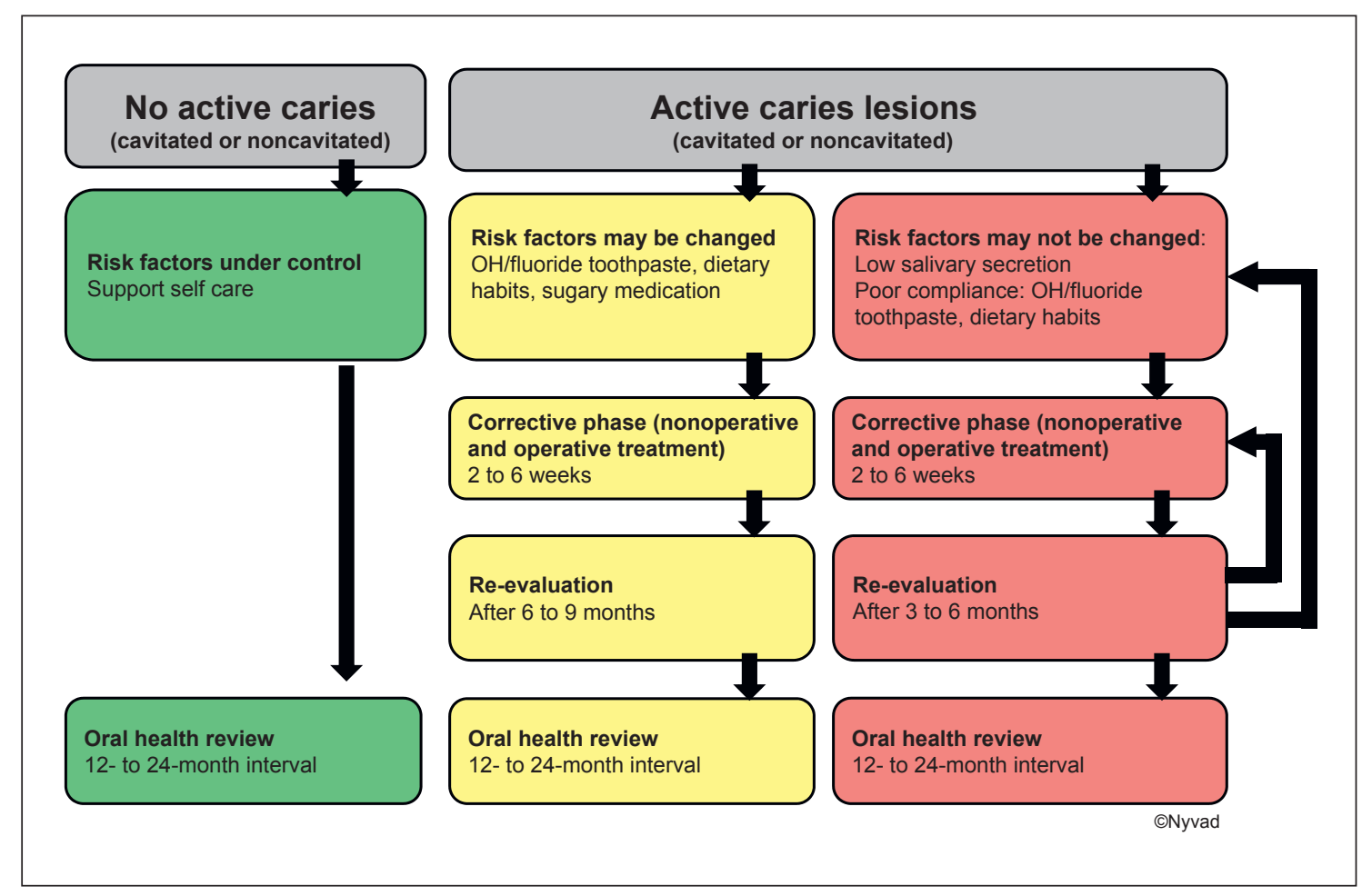

Fig. 3. Guideline for categorizing patients into caries activity and caries risk status and for setting the recall interval for caries control [Nyvad and Kidd, 2015]. For detailed description, see text. OH, oral hygiene. Reproduced with permission of Wiley Blackwell.

The clinical benefit of using the Nyvad criteria for the purpose of caries management gains further support by the ability of the criteria to identify individuals with a high risk for future caries progression. Previous approaches to the identification of cases with increased caries risk have mainly relied on various caries risk assessment tools such as the Cariogram or CAMBRA (caries management by risk assessment). However, the prognostic value of such tools may be modest [Tellez et al., 2013]. The single best risk factor for new caries appears to be past caries experience [Hausen, 1997]. Unfortunately, this factor has limited prognostic value in patients with multiple restorations and in patients who have already mastered caries control. Modern evidence-based protocols for caries management should, therefore, start by identifying patients at risk of future caries by assessing caries lesion activity (Fig. 3) [Nyvad and Kidd, 2015]. If there are no active lesions at the time of examination, the patient is advised to maintain self-care (green lane patient). If the patient reveals active caries lesions, the caries-promoting factors should be evaluated for the actual patient and be modified by appropriate interventions along with nonop- erative and operative treatment of the active lesions (yellow lane patient). If risk factors cannot be modified (such as in patients with decreased salivary secretion), intensified professional interventions are needed (red lane patient) [Nyvad and Kidd, 2015]. Such an approach for controlling dental caries was put into service in clinical practice in Denmark by the Danish Health Authority [2013]. Yet, additional longitudinal studies are required to determine the potential superiority of the Nyvad criteria as a screening tool for caries management strategies compared to conventional risk-based approaches.

\section{Planning and Organization of Dental Public Health Services}

Dental public health workers may benefit considerably from having access to data about the severity and activity of dental caries in their community to gain an overview of the disease status and treatments needed. Clinical examinations based on the Nyvad criteria automatically generate such data. Hence, rather than merely obtaining information about the number of cavitated and noncavitated caries lesions, the Nyvad criteria provide additional 
information about the amount of active noncavitated lesions indicated for nonoperative treatment [Machiulskiene et al., 1998, 2009]. Based on such knowledge, nonoperative and operative treatments may easily be shared among different members of the dental team to ensure that oral health services are delivered in a cost-effective manner.

\section{Research Applications}

Over the years, the Nyvad criteria have proven their unique potential as a research tool for exploring transition patterns of caries lesions in response to various caries-controlling interventions. This property goes beyond all traditional caries classification systems validated against lesion depth only, including systems that do not differentiate between active and inactive stages of caries at the noncavitated level (e.g., ICDAS II). A convincing example is the previously reported clinical trial of supervised brushing with fluoride toothpaste [Machiulskiene et al., 2002] showing that fluoride inhibited lesion progression at all stages of caries formation (preventive effect) while at the same time promoting lesion regression (therapeutic effect) [Nyvad et al., 2003]. Survival time analyses of the lesion transitions disclosed that the therapeutic effect was much more prominent than the preventive effect [Baelum et al., 2003]. Remarkably, when comparing these data with the pattern of lesion transitions in a parallel trial of chewing sugar-free gums [Machiulskiene et al., 2001], it was found that the preventive effect of chewing sugar-free gum was smaller than when brushing with fluoride toothpaste, and there was hardly any recovery/therapeutic effect of gum chewing on the active lesions [Baelum et al., 2003; Nyvad et al., 2005]. Collectively, these observations suggest that the pattern of lesion transitions reflect the nature of the caries-controlling intervention, a phenomenon that should be further exploited in future caries-preventive trials using the Nyvad criteria.

The ability of the Nyvad criteria to reflect differences in the anticaries effect of a 500- versus a 1,100-ppm fluoride toothpaste was convincingly shown in a clinical trial comprising children of different caries activity status [Lima et al., 2008]. The effect of the low-fluoride toothpaste was similar to that of the conventional toothpaste in caries-inactive children. However, in children with active caries lesions, the low-fluoride paste was less effective than the $1,000-p p m$ paste in controlling the progression of lesions.

Finally, the Nyvad criteria are also suitable for demonstrating the effect of fluoride on caries activity and caries severity scores in cross-sectional and observational studies. Children born and raised in a water-fluoridated area (1.1 ppm F) had more inactive lesions and fewer fillings than children of similar age living in an area with low concentrations of fluoride in the drinking water $(0.3 \mathrm{ppm} \mathrm{F})$ [Machiulskiene et al., 2009]. A recent observational study of children living in a water-fluoridated community (0.7 ppm F) showed a low proportion of noncavitated lesions progressing to cavitation over 2 years [Cabral et al., 2017]. Analysis of the lesion transitions revealed that the low number of transitions to cavitation could be explained by $45 \%$ of the active noncavitated lesions arresting or regressing to sound while at the same time $85 \%$ of the inactive noncavitated lesions remained stable or regressed to sound. Clearly, use of the Nyvad criteria in the above studies added new information about caries lesion transition patterns at the noncavitated level that could not have emerged had the diagnostic criteria not included lesion activity assessment.

\section{Why Should Clinicians and Researchers Use the Nyvad Criteria for Caries Assessment?}

The Nyvad caries diagnostic criteria enable monitoring of the progression stage and activity of caries lesions simultaneously, which is of particular importance in lowcaries populations. The criteria consist of 10 codes including active and inactive stages of increasing severity of lesion formation, as well as separate codes for sound surfaces, fillings, and secondary caries. The straightforward design of the criteria using a 1-digit coding system and its ease of use [Séllos and Soviero, 2011] is appealing to clinicians and researchers compared to the ICDAS criteria, for example, that have been described as cumbersome to report and analyze [de Amorim et al., 2012].

The Nyvad criteria are reliable within and between trained examiners, and they have construct and predictive validity. The criteria, therefore, fulfill all the formal requirements for a robust caries lesion classification. In particular, the predictive validity makes the criteria superior to other current lesion classifications by allowing identification of individuals at increased risk for caries progression and by indicating individual lesions in need of professional treatment (nonoperative or operative).

Studies applying the Nyvad criteria have consistently confirmed the effect of fluoride on active caries processes in cross-sectional/observational studies and in clinical trials. Because of the ability of the criteria to reflect caries lesion dynamics at both the cavitated and noncavitated 
levels of lesion formation, the criteria are highly recommended for studies of the therapeutic effect of caries-controlling interventions. To the best of our knowledge, no other caries lesion classification has been able to mirror the de- and remineralization processes in caries to a similar extent. The ICDAS criteria, because of their rationale based on lesion depth, are capable of reflecting the stage of lesion development only [Ferreira Zandoná et al., 2012].

Finally, the description of the Nyvad caries lesion classification has remained unchanged since its introduction in 1999. Meanwhile, other lesion classifications have incorporated "modifications" [ICDAS II, 2009; ICCMS ${ }^{\mathrm{TM}}$, 2014] or even copied [Guedes et al., 2014, 2015] the Nyvad criteria into their protocols. However, the combination of elements from different lesion classifications does not guarantee validity of the resulting merged criteria. In fact, adoption of the Nyvad classification into the ICDAS protocol is redundant because the Nyvad criteria already cover all relevant aspects of caries lesion severity and activity.

\section{Disclosure Statement}

None of the authors has any conflict of interest.

\section{Author Contributions}

B.N. drafted the paper. V.B. critically reviewed the paper. Both authors gave final approval.

\section{References}

Ando M, Ferreira-Zandoná AG, Eckert GJ, Zero DT, Stookey GK: Pilot clinical study to assess caries lesion activity using quantitative lightinduced fluorescence during dehydration. J Biomed Opt 2017;22:035005.

Baelum V, Heidmann J, Nyvad B: Dental caries paradigms in diagnosis and diagnostic research. Eur J Oral Sci 2006;114:263-277.

Baelum V, Machiulskiene V, Nyvad B, Richards A, Vaeth M: Survival modelling of caries lesion transitions in community intervention trials of preventive interventions. Community Dent Oral Epidemiol 2003;31:252-260.

Braga MM, Ekstrand KR, Martignon S, Imparato JCP, Ricketts DNJ, Mendes FM: Clinical performance of two visual scoring systems in detecting and assessing activity status of occlusal caries in primary teeth. Caries Res 2010;44: 300-308.

Cabral RN, Freitas E, Soviero VM, Nyvad B, Leal SC: Caries progression in a cohort of Brazilian schoolchildren assessed by the Nyvad criteria. Caries Res 2017;51:350 (abstract).

Danish Health Authority: National klinisk retningslinje - Fastlæggelse af intervaller mellem diagnostiske undersøgelser i tandplejen. 2013 (version 2: July 2016), https://www.sst.dk/da/ udgivelser/2016/nkr-tandplejen.

de Amorim RG, Figueiredo MJ, Leal SC, Mulder J, Frencken JE: Caries experience in a child population in a deprived area of Brazil, using ICDAS II. Clin Oral Investig 2012;16:513520.

Fejerskov O, Thylstrup A, Larsen MJ: Rational use of fluorides in caries prevention. A concept based on possible cariostatic mechanisms. Acta Odontol Scand 1981;39:241-249.
Ferreira Zandoná A, Santiago E, Eckert GJ, Katz BP, Pereira de Oliveira S, Capin OR, Mau M, Zero DT: The natural history of dental caries lesions: a 4-year observational study. J Dent Res 2012;9:841-846.

Guedes RS, Piovesan C, Ardenghi TM, Emmanuelli B, Braga MM, Ekstrand KR, Mendes FM: Validation of visual caries activity assessment: a 2-yr cohort study. J Dent Res 2014; 93(suppl 7):101S-107S.

Guedes RS, Piovesan C, Ardenghi TM, Emmanuelli B, Braga MM, Ekstrand KR, Mendes FM: Corrigendum. J Dent Res 2015;94:1784.

Hansen NV, Nyvad B: Non-operative control of cavitated approximal caries lesions in primary molars: a prospective evaluation of cases. J Oral Rehabil 2017;44:537-544.

Hausen H: Caries prediction - state of the art. Community Dent Oral Epidemiol 1997;25: $87-96$.

Hausen H, Kärkkäinen S, Seppä L: Application of the high-risk strategy to control dental caries. Community Dent Oral Epidemiol 2000;28: 26-34.

Hausen H, Seppä L, Poutanen R, Niinimaa A, Lahti S, Kärkkäinen S: Noninvasive control of dental caries in children with active initial lesions. Caries Res 2007;41:384-391.

International Caries Detection and Assessment System (ICDAS) Coordinating Committee: Rationale and Evidence for the International Caries Detection and Assessment System (ICDAS II). Appendix. Criteria Manual. 2009, https://www.icdas.org/uploads/ICDAS\%20 Criteria\%20Manual\%20Revised\%202009_2. pdf (accessed February 3, 2017).
International Caries Classification and Management system. ICCMS ${ }^{\mathrm{TM}}$ Guide for Practitioners and Educators. 2014, https://www.icdas. org/uploads/ICCMS-Guide_Full_Guide_ With_Appendices_UK.pdf (accessed February 3,2017 ).

Ismail AI, Brodeur J-M, Gagnon P, Payette M, Picard D, Hamalian T, Oliver M, Eastwood BJ: Prevalence of non-cavitated and cavitated carious lesions in a random sample of 7-9-year-old schoolchildren in Montreal, Quebec. Community Dent Oral Epidemiol 1992;20:250-255.

Ismail AI, Sohn W, Tellez M, Amaya A, Sen A, Hasson H, Pitts NB: The International Caries Detection and Assessment System (ICDAS): an integrated system for measuring dental caries. Community Dent Oral Epidemiol 2007;35:170-178.

Last JM (ed): A Dictionary of Epidemiology, ed 4. Oxford, Oxford University Press, 2001.

Lima TJ, Ribeiro CCC, Tenuta LMA, Cury JA: Low-fluoride dentifrice and caries lesion control in children with different caries experience: a randomized clinical trial. Caries Res 2008;42:40-50.

Machiulskiene V, Nyvad B, Baelum V: Prevalence and severity of dental caries in 12-year-old children in Kaunas, Lithuania, 1995. Caries Res 1998;32:175-180.

Machiulskiene V, Nyvad B, Baelum V: Caries preventive effect of sugar-substituted chewing gums. Community Dent Oral Epidemiol 2001;29:278-288.

Machiulskiene V, Nyvad B, Baelum V, Fejerskov O: Prevalence and extent of dental caries, dental fluorosis and developmental defects in Lithuanian teenage populations with different fluoride exposure. Eur J Oral Sci 2009;117: 154-160. 
Machiulskiene V, Richards A, Nyvad B, Baelum V: Prospective study of the effect of postbrushing rinsing behaviour on dental caries. Caries Res 2002;36:301-307.

Nyvad B: Diagnosis versus detection of caries. Caries Res 2004;38:192-198.

Nyvad B, Fejerskov O: Active root surface caries converted into inactive caries as a response to oral hygiene. Scand J Dent Res 1986;94:281284.

Nyvad B, Fejerskov O: An ultrastructural study of bacterial invasion and tissue break-down in human experimental root surface caries. J Dent Res 1990;69:1118-1125.

Nyvad B, Fejerskov O: Assessing the stage of caries lesion activity on the basis of clinical and microbiological examination. Community Dent Oral Epidemiol 1997;25:69-75.

Nyvad B, Fejerskov O: The caries control concept; in Fejerskov O, Nyvad B, Kidd E (eds): Dental Caries: The Disease and Its Clinical Management, ed 3. Oxford, Wiley Blackwell, 2015, pp 235-243.

Nyvad B, Kidd EAM: The principles of caries control for the individual patient; in Fejerskov $\mathrm{O}$, Nyvad B, Kidd E (eds): Dental Caries: The Disease and Its Clinical Management, ed 3. Oxford, Wiley Blackwell, 2015, pp 303-320.
Nyvad B, Machiulskiene V, Baelum V: Reliability of a new caries diagnostic system differentiating between active and inactive caries lesions. Caries Res 1999;33:252-260.

Nyvad B, Machiulskiene V, Baelum V: Construct and predictive validity of caries diagnostic criteria assessing lesion activity. J Dent Res 2003; 82:117-122.

Nyvad B, Machiulskiene V, Baelum V: The Nyvad criteria for assessment of caries lesion activity; in Stookey G (ed): Proceedings of the 7th Indiana Conference, Indiananapolis. Indiana. Clinical Models Workshop: Remin-Demin, Precavitation, Caries. Indianapolis, Indiana University School of Dentistry, 2005, pp 99116.

Nyvad B, Machiulskiene V, Fejerskov O, Baelum V: Diagnosing dental caries in populations with different levels of dental fluorosis. Eur J Oral Sci 2009;117:161-168.

Nyvad B, Machiulskiene V, Soviero V, Baelum V: Visual/tactile caries diagnosis; in Fejerskov $\mathrm{O}$, Nyvad B, Kidd E (eds): Dental Caries: The Disease and Its Clinical Management, ed 3. Oxford, Wiley Blackwell, 2015, pp 191-210.
Nyvad B, ten Cate JM, Fejerskov O: Arrest of root surface caries in situ. J Dent Res 1997;76: 1845-1853.

Séllos MC, Soviero VM: Reliability of the Nyvad criteria for caries assessment in primary teeth. Eur J Oral Sci 2011;119:225-231.

Tellez M, Gomez J, Pretty I, Elwood R, Ismail AI: Evidence on existing caries risk assessment systems: are they predictive of future caries? Community Dent Oral Epidemiol 2013;41: 67-78.

ten Cate JM, Featherstone JDB: Physicochemical aspects of fluoride-enamel interactions; in $\mathrm{Fe}$ jerskov O, Ekstrand J, Burt BA (eds): Fluoride in Dentistry. Copenhagen, Munksgaard, 1996, pp 252-272.

Thylstrup A, Bruun C, Holmen L: In vivo caries models - mechanisms for caries initiation and arrestment. Adv Dent Res 1994;8:144-157.

Tikhonova SM, Feine JS, Pustavoitava NN, Allison PJ: Reproducibility and diagnostic outcomes of two visual-tactile criteria used by dentists to assess caries lesion activity: a crossover study. Caries Res 2014;48:126-136.

WHO: Oral Health Surveys: Basic Methods, ed 4. Geneva, World Health Organization, 1997. 